\title{
Severe unconjugated hyperbilirubinaemia with infradiaphragmatic total anomalous pulmonary venous connection
}

\author{
Michael Burch, Umesh Dyamenahalli, Ian D Sullivan
}

\begin{abstract}
An 8 day old girl with infradiaphragmatic total anomalous pulmonary venous connection presented with severe unconjugated hyperbilirubinaemia; exchange transfusion resulted in haemodynamic deterioration. Hyperbilirubinaemia is a rare complication of this condition, however exchange transfusion should be avoided. The diagnosis can be made on the neonatal unit by ultrasound demonstration of dilated hepatic portal veins.

(Arch Dis Child 1993; 68: 608-609)
\end{abstract}

The typical presentation of infradiaphragmatic total anomalous pulmonary venous connection is with tachypnoea and cyanosis in the early neonatal period because of obstruction to pulmonary venous drainage in the liver. We report a neonate who presented with severe unconjugated hyperbilirubinaemia.

\section{Case report}

A girl was born to non-consanguinous Asian parents at full term with a birth weight of $2600 \mathrm{~g}$. After a routine neonatal examination she was discharged home at 24 hours. Breast feeding was established, but tachypnoea developed on day 3. Jaundice was noted by the attending midwife on day 4 and was felt to be physiological. It did not improve, however, and serum bilirubin concentration on day 7 was $615 \mu \mathrm{mol} / \mathrm{l}$ ( $565 \mu \mathrm{mol} / \mathrm{l}$ unconjugated). After emergency admission to a neonatal unit an infection screen, including a lumbar puncture, was performed but no evidence of acute infection was documented. Both mother and infant were blood group B positive and a direct Coombs test was negative. In view of the severity of the hyperbilirubinaemia, with the associated risk of kernicterus, exchange transfusion was commenced. The exchange transfusion was performed in the standard way, with peripheral venous and arterial cannulas using a 'push/pull' method with aliquots of $5 \mathrm{ml}$. During the first transfusion marked deterioration occurred, with severe hypoxia, hypotension, poor peripheral perfusion, and acidosis. The child improved after the institution of positive pressure ventilation. As the serum bilirubin had decreased only to $400 \mu \mathrm{mol} / \mathrm{l}$ (fig 1) a second exchange transfusion was performed. Again, similar severe haemodynamic deterioration occurred. As she remained hypoxic breathing $100 \%$ oxygen (arterial oxygen tension increasing from $8.4 \mathrm{kPa}$ breathing $40 \%$ oxygen to $14.9 \mathrm{kPa}$ ) she was transferred to a cardiology unit on day 8 , where echocardiography demonstrated infradiaphragmatic total anomalous pulmonary venous connection with marked portal venous congestion (fig 2). Emergency surgical correction was performed. There was pulmonary hypertension immediately after the operation but the postoperative period was otherwise uncomplicated. Hyperbilirubinaemia declined steadily, and at discharge on day 14 serum bilirubin concentration was $66 \mu \mathrm{mol} / \mathrm{l}$ (fig 1). At 6 week follow up she was thriving and serum bilirubin was $<12 \mu \mathrm{mol} / 1$.

Investigations taken before cardiopulmonary bypass included: asparginine transaminase activity 34 IU/l (normal 10 to 60 ), alkaline phosphatase activity $231 \mathrm{IU} / 1$ (normal 65-265), albumin concentration $40 \mathrm{~g} / \mathrm{l}$, blood film normal, haemoglobin electrophoresis normal, sickle screen negative, glucose-6phosphate dehydrogenase activity $11 \cdot 1 \mathrm{IU} / \mathrm{g}$ haemoglobin (normal 6.8-9.3), hepatitis B antigen negative, toxoplasma and cytomegalovirus antibodies not raised, thyroid function tests normal, coagulation screen normal, prothrombin time $15 / 12$, and no urinary reducing

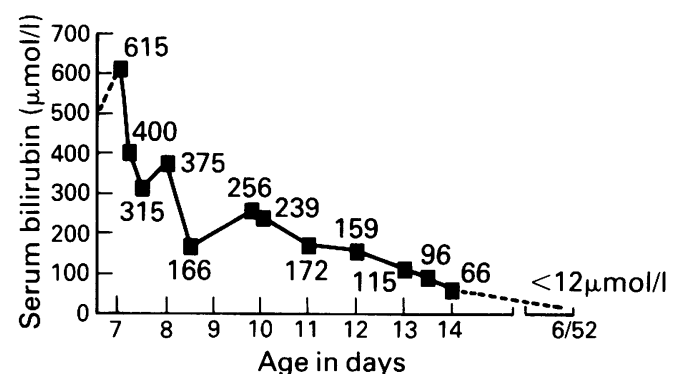

Figure 1 Serum bilirubin concentration in relation to age (days). Exchange transfusion was performed when bilirubin was $615 \mu \mathrm{mol} / \mathrm{l}$ (day 7) and again when 400 $\mu \mathrm{mol} / \mathrm{s}$.

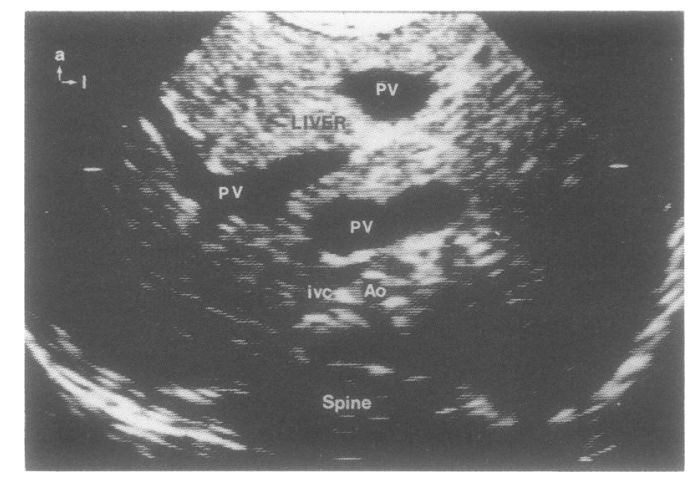

Figure 2 Hepatic ultrasound demonstrating enlarged portal veins $(P V) ; i v c=$ inferior vena cava, $A o=$ aorta 
substances. Postoperative liver ultrasound was normal.

\section{Discussion}

Severe hyperbilirubinaemia as the presenting feature of total anomalous pulmonary venous drainage was not reported in reviews which examined clinical features of the condition. ${ }^{12}$ Hepatic ischaemia is more important than hepatic venous congestion in causing neonatal hyperbilirubinaemia in association with congenital heart disease. ${ }^{34}$ However, the normal liver function tests and postoperative liver ultrasound in our patient make major ischaemic damage unlikely. In infradiaphragmatic total anomalous pulmonary venous connection the pulmonary venous drainage is almost always to the portal venous system. After the ductus venosus has closed, the entire pulmonary blood flow must traverse hepatic sinusoids to reach hepatic veins and inferior vena cava. It is not surprising that the sinusoidal distension might be associated with hepatocyte dysfunction, and consequently exaggeration of physiological jaundice. Thus, some degree of jaundice would be expected with infradiaphragmatic total anomalous pulmonary venous drainage, but severe jaundice is a rare presentation of the condition. It should, however, be considered in the differential diagnosis as distended portal veins can be readily demonstrated by liver ultrasound in the neonatal unit. Other causes of distended portal veins such as the Budd-Chiari syndrome are uncommon in the newborn, and would usually be associated with distended superficial abdominal veins.
It would be helpful to exclude infradiaphragmatic total anomalous pulmonary venous drainage before undertaking an exchange transfusion as colloid load is poorly tolerated in the condition, worsening of pulmonary oedema being an inevitable consequence. The exchange transfusion in the case described was performed in the standard way, yet appeared to coincide with a haemodynamic deterioration. Clearly it is impossible to attribute the deterioration entirely to the exchange, but we feel such an intervention is contraindicated in this anomaly. A greater deterioration would have been anticipated if the umbilical vein had been used, and the cannula had not passed through the liver. If the diagnosis is established on ultrasound early referral for cardiac surgery is appropriate even if jaundice is severe, 'exchange transfusion' is effectively performed by cardiac surgery.

In conclusion, infradiaphragmatic total anomalous pulmonary venous drainage is worthy of consideration in the differential diagnosis of neonatal jaundice, and can easily be excluded by hepatic ultrasound. Severe jaundice, as in this case, is rare but in such cases exchange transfusion should be undertaken with extreme caution, or perhaps not at all.

We are grateful to Dr P Clayton for advice regarding the investigation of hyperbilirubinaemia.

1 Bonham Carter RE, Capriles M, Noe Y. Total anomalous pulmonary venous drainage: a clinical and anatomical study of 75 children. Br Heart $\mathcal{f} 1969 ; 31: 45-51$.

2 Gathman GE, Nadas AS. Total anomalous pulmonary venous connection: clinical and physiological observations of 75 pediatric patients. Circulation 1970; 42: 143-54.

3 Weinberg AG, Bolande RP. The liver in congenital heart disease. Am f Dis Child 1970; 119: 391-4.

4 Shiraki K. Hepatic cell necrosis in the newborn. Am $f$ Dis Child 1970; 119: 395-400. 